\title{
Space Telescope Sensitivity and Controls for Exoplanet Imaging
}

\author{
Richard G. Lyon*a, Mark Clampin ${ }^{\mathrm{a}}$ \\ ${ }^{a}$ NASA Goddard Space Flight Center, Greenbelt, MD, USA 20771
}

\begin{abstract}
Herein we address design considerations and outline requirements for space telescopes with capabilities for high contrast imaging of exoplanets. The approach taken is to identify the span of potentially detectable Earth-sized terrestrial planets in the habitable zone of the nearest stars within 30 parsecs and estimate their inner working angles, flux ratios, SNR, sensitivities, wavefront error requirements and sensing and control times parametrically versus aperture size. We consider 1,2, 4,8 and 16-meter diameter telescope apertures. The achievable science, range of telescope architectures, and the coronagraphic approach are all active areas of research and are all subject to change in a rapidly evolving field. Thus, presented is a snapshot of our current understanding with the goal of limiting the choices to those that appear currently technically feasible. We describe the top-level metrics of inner working angle, contrast and photometric throughput and explore how they are related to the range of target stars. A critical point is that for each telescope architecture and coronagraphic choice the telescope stability requirements have differing impacts on the design for openversus closed-loop sensing and control.
\end{abstract}

Keywords: Telescope, coronagraphy, exoplanets, wavefront sensing, wavefront control

\section{INTRODUCTION}

High contrast exoplanet imaging refers to numerous variations of coronagraphy and occulters whereby the starlight is suppressed relative to the planet light, thereby increasing the planet-to-star contrast. Contrast, as defined herein, is expressed as a ratio of the of the star's luminosity to planet's luminosity. A coronagraph operating at a contrast of $10^{10}$ would imply that a star and planet differing by 10 orders of magnitude in luminosity would be detectable in the focal plane. An ideal coronagraph would suppress all the starlight leaving only the planet's light. The contrast that a given coronagraph operates at is a function of the angular separation of the planet-to-star since a planet with a large angular separation from its parent star sees a natural reduction in the starlight due it being concentrated into an Airy disk point spread function (Figure-1 left). The planet-to-star angular separation at which the contrast falls below a required value is known as the inner working angle (IWA). Thus planets at or outside the IWA are considered detectable, while those in 
the IWA are not. However, there is not a definitive break between inside and outside the IWA since the detectability is often a graceful function of the IWA (Figure-1 right). The outer working angle (OWA) is the largest separation angle at which a planet could be detected and this is generally limited by the field-of-view of the optics and/or the number and density of actuators of the deformable mirrors within the coronagraph.

Light collected by the aperture is absorbed, diffracted and/or scattered. It thus absorbs and spreads the focal plane planet light out over a larger region, and scatters starlight into the region of the planet; these effects lower the contrast. Throughput is generally lower for high contrast imaging systems than for simple imaging systems. This is due to the larger number of optics and the introduction of focal planet masks (occulting masks) and pupil plane (Lyot) masks. Ideally the star-and planet-light throughput would be zero and unity respectively. However this is not generally true in practice due to a host of effects that will be described.

The target star, if we assume it has one or more planets, would likely have dust and debris at or near the equatorial plane of the star; this contributes straylight known as exozodiacal light (exoZodi). Additionally dust in our solar system also contributes straylight known as local zodiacal light (Zodi).

The target star is generally relatively close to our solar system, from $1-30$ parsecs, and thus is likely to have background stars and galaxies within the field-of-view that could initially be misinterpreted as planets. Relative motion and/or spectral differences can be used as discriminators. Additionally since a planet orbits its star, conditions would not always be favorable to detection since it may be too close to the star, or at a phase angle or inclination angle where little, or no, light is reflected from the planet towards the telescope. A single observation can only determine if a planet exists, but not whether it does not exist. Thus a given star system may have to be observed multiple times to definitively determine whether a planet exists. This is known as "completeness" (Brown (2004, Brown (2005, Brown and Soummer, (2010)). Ensuring completeness to a given level links the number of observations for each star system, to overall mission lifetime.

The signal-to-noise ratio (SNR) is the ratio of the collected planetary photons to the integrated noise contribution, which includes stellar leakage from diffraction, imperfect optics, occulting masks, Lyot stops, straylight, pointing jitter, finite size of target star, thermal and vibration induced optical instabilities, photon noise, read noise, dark current noise, and 
out of field scatter. The SNR is a function of contrast, angular separation, and time. Solving for the time to a given SNR yields a viable sensitivity metric, e.g. solving for the time to a SNR $=5$ (5-sigma detection) at the IWA.

Contrast, IWA, OWA, and optical throughput are purely instrumental (telescope + coronagraph) parameters whereas SNR and sensitivity depends on the target star, angular separation of planet to star as well as the instrument's performance, thus the discovery space of potential science targets plays a large role in defining the required telescope and coronagraph architecture.

The purpose of an exoplanet high contrast system is to detect and characterize exosolar planets. This problem is manifestly difficult due to typical terrestrial planets being $\sim 10^{10}$ times dimmer than the parent star in reflected light and at angular separations as close as a few 10's of milli-arcseconds. In designing a high contrast imaging system, performance considerations have to include: aperture size, diffracting structure within the aperture, optical surface quality, optical stability, polychromatic effects, polarization, sensing and control approach and control times relative to drift rates, and pointing, and post-processing such as point spread function and background subtraction.

High contrast imaging for exoplanets is not a new problem. The Hubble Space Telescope's (HST) original instrument complement contained a coronagraph, the Faint Object Camera (FOC) f/288 coronagraphic mode. However, few images were collected since HST's primary mirror conic constant rendered it impractical for coronagraphy. Subsequent HST instruments such as NICMOS, STIS and ACS/HRC had coronagraphs within them. However, contrasts corresponding to $10^{-9}$ to $10^{-10}$ suppression of the central star could only be achieved at significant OWA (e.g. Kalas et al 2008). If a future servicing mission were available for the Hubble Space Telescope (HST) then an area where a new HST scientific instrument could have a high impact on exoplanet science is with a high contrast instrument (Lyon, et. al. 2010). The James Webb Space Telescope (JWST) also will have significant high contrast imaging capabilities (Clampin et al. (2007)).

\section{MAPPING OF SCIENCE REQUIREMENTS TO TELESCOPE}

\subsection{Candidate Stars}

A database (HIP30), from the Hipparcos mission (Schneider 2011, Turon et. al. 1995), of 2350 stars out to 30 parsecs has become a standard for designing mission architectures for direct imaging exoplanet missions. The HIP30 catalog has 
been culled to a shorter list of 575 viable candidates, searchable for exoplanets by assuming that for each star a prospective planet exists at the orbital distance where liquid water could exist, i.e. in the habitable zone (HZ). The inner edge (water boils off and/or runaway greenhouse effect) of this zone is the inner $\mathrm{HZ}(\mathrm{iHz}$ ) and outer edge (where water remains in ice phase) is the outer $\mathrm{HZ}(\mathrm{oHZ})$ for terrestrial planets. We define a average $\mathrm{HZ}$ (aveHZ) as aveHZ = $(1 / 2)(\mathrm{iHZ}+\mathrm{oHZ})$ and use this as its nominal value.

Figure-2 plots the 575 candidates versus distance from out solar system where the "All Stars" are the 2350 stars in the database and "Candidates" are the selected 575 candidate stars. The 5 other curves on show the stars by spectral classes based on effective blackbody temperature, $\mathrm{M}(<3,500 \mathrm{~K}), \mathrm{K},(3,500-5,000 \mathrm{~K}), \mathrm{G}(5,000-6,000 \mathrm{~K}), \mathrm{F}(6,000-7,500 \mathrm{~K})$ and $\mathrm{A}$ $(7,500-10,000 \mathrm{~K})$. The number to the right of the spectral class in the legend shows the number of stars for each spectra class. There are 24 stars labeled as U-stars (for unknown) that have no listed spectral class in the database. G-stars are similar in spectral class to our own Sun. Overall there are 163 M, 219 K 124 G, 27 F and 18 A candidates. If a terrestrial planet existed within the $\mathrm{HZ}$ then based on the stars luminosity and spectral class a region around the star can be mapped to an angular separation of the planet from its parent star versus distance to the star as shown in Figure-3. The angular scale of the $\mathrm{HZ}$ is an important consideration in the sizing of the telescope aperture and sets the IWA for high contrast imaging. It is unlikely that all candidates will have a planet and the probability that a given planet has a terrestrial planet is known as $\eta_{\text {EARTH }}$. Various estimates are available for as $\eta_{\text {EARTH }}$ and NASA's ongoing Kepler mission and ESA's ongoing COROT mission, will likely allow a more refined estimate in the near future.

Scatter, diffraction, straylight and stability of the sensing and control limit the IWA. An approach limited to for example IWA $=2 \lambda / D$ requires accurate sensing and control of wavefront and amplitude spatial frequencies at $\sim 2$ cycles per aperture, i.e. of periods on the order of $1 / 2$ the diameter. This is due to the diffraction integral, in the small angle approximation, mapping periodic wavefront errors at $\mathrm{N}$ cycles per aperture to localized speckle in the focal plane at $\mathrm{N}$ $\lambda / D$. Thus approaches operating at small inner working angle require more demanding tolerances at low spatial frequencies, i.e. for points on the primary mirror that are physically further apart rand thereby more difficult to sense and control. While $4 \lambda / \mathrm{D}$ is considered as being conservative (Trauger \& Traub (2007)) and $1 \lambda / \mathrm{D}$ as being considered very aggressive, it is expected that in the nearer term, while still aggressive, $2 \mathrm{ND}$ is more reasonable and exoplanets from the ground have been imaged at this IWA (Serabyn et. al. (2010)). Thus, herein we assume a coronagraphic instrument with 
an IWA of $2 \lambda / \mathrm{D}$ where $\lambda$ is the wavelength of visible band reflected planetary light $(\lambda=550 \mathrm{~nm})$ and $\mathrm{D}$ is the diameter of the aperture.

The $\mathrm{HZ}$ is mapped to an aperture of a specific diameter, by setting the IWA to the mean $\mathrm{HZ}, \mathrm{IWA}=2 \lambda / D=\mathrm{HZ}$ or $D=2 \lambda / H Z$, and the integral number of candidates at or outside an IWA $=2 \lambda / \mathrm{D}$ versus aperture diameter is plotted in Figure-4. Scaling to a different IWA is accomplished by shifting the abscissa by the same factor, e.g. for an IWA $=4$ $\lambda / \mathrm{D}$ would require the abscissa (on a $\log$ scale) to shifted to the left such that on Figure- 4 a meter aperture is shifted to a $1 / 2$ meter etc and thus the number of candidates would decrease. From Figure-4 it is seen that a 120-meter diameter telescope operating at IWA $=2 \lambda / \mathrm{D}$ would be required to assess all the candidate stars out to 30 parsecs. This excessively large aperture is driven by the M-stars that are colder and have peak luminosity in the NIR requiring that a planet in in the $\mathrm{HZ}$ be at closer angular separation for liquid water to exist.

A 120 -meter aperture telescope operating at IWA $=2 \lambda / \mathrm{D}$ is clearly difficult for a space mission but filtering out those M-stars requiring close IWA allows for more reasonable sized aperture as shown in Table-1. If $\eta_{\text {EARTH }}$ is assumed to be $20 \%$ then the number of candidates would decrease by a factor of 5 . Jovian (Jupiter analogues) planets could also be searched for and would generally result in a larger IWA.

\subsection{Planet-to-Star Luminosity Ratio and Aperture Flux}

The aperture sizing is based purely on inner working angle but the aperture collecting area is the primary consideration for signal-to-noise (SNR) ratio and sensitivity. Detector integration time could become prohibitive for some candidates due to low planetary photon count rates in visible light. A photometric model can be used to assess the photon count rates for contrast, SNR and sensitivity calculations.

The luminosity ratio (Charbonneau et.al. 1999, Winn 2011) of the reflected planet light to emitted starlight is given by:

$$
\text { Luminosity Ratio }=\frac{L_{P}}{L_{S}}=\alpha\left(\frac{R_{P}}{R_{d}}\right)^{2} \phi(\beta)
$$


$L_{P}$ and $L_{S}$ are the luminosities of the planet and star respectively, $\alpha$ is the planets geometric albedo, $R_{p}$ is the radius of the planet and $R_{d}$ is the planets radial distance from the star. $\phi(\beta)$ is the orbital phase function given by $\phi(\beta)=\frac{\sin \beta+(\pi-\beta) \cos \beta}{\pi}$ where $\beta \in[0, \pi]$ is the phase angle and where $\cos \beta=-\sin (i) \sin (2 \pi \Phi)$ where $i \in\left[0, \frac{\pi}{2}\right]$ is the inclination angle. An inclination angle of 0 implies the star system is seen face-on, and an inclination of $\pi / 2$ implies the star system is seen edge-on. The orbital phase is given by $\Phi \in[0,1]$ and for an inclination angle of 0 then $\phi(\beta) \approx 0.32$ independent of phase angle. At an inclination angle of 90 degrees the phase function reaches a maximum of $\sim 0.32$ and a value of 0.32 is used throughout, i.e. we assumed the best viewing conditions. The geometric albedo of Earth is 0.367 and this is also assumed throughout. The phase function is 0.32 for the point of longest elongation and this is not necessarily where the planet is brightest but where planet is visible (Charbonneau 1999). The radius of the Earth is $6378.1 \mathrm{~km}$ and the Sun-Earth distance is $149 \times 10^{6} \mathrm{~km}$, yielding an Earth/Sun luminosity ratio of $2.2 \times 10^{-10}$. This value can be used to scale the luminosity ratio of an assumed Earth-sized terrestrial planet at the mean HZ around stars within the database via:

$$
\frac{L_{P} / L_{S}}{L_{\text {EARTH }} / L_{S U N}}=\frac{\alpha_{P}}{\alpha_{E A R T H}}\left(\frac{R_{P}}{R_{P-E A R T H}}\right)^{2}\left(\frac{R_{d-E A R T H}}{R_{d}}\right)^{2}
$$

where $L_{E A R T H} / L_{S U N}=2.2 \times 10^{-10}$ is the luminosity ratio of Earth to the Sun and $L_{P} / L_{S}$ is the luminosity ratio of the planet to star. The albedo and the radius of the planet are both not generally known before an observation and we assume they are both the same as for Earth to arrive at:

$$
\frac{L_{P}}{L_{S}}=2.2 \times 10^{-10}\left(\frac{R_{d-E A R T H}}{R_{d}}\right)^{2}
$$


The radial distance for Earth from our Sun is 1-AU, and as expected, the luminosity ratio scales inversely proportional to the square of the distance from the star. The inverse square is a consequence of assuming a the fixed Earth diameter planet, independent of how it formed or distance from its parent star, and, that its albedo is also constant; thus it's subtended solid angle as seen from it's parent star scales inversely as the square of its distance from its parent star. The Earth-sized planet is a tenuous assumption since terrestrial planets are likely to span a range of sizes, masses, albedos inclination and phase angles; it is however a reasonable assumption as a starting point for defining a range of potential telescope architectures.

Figure-5 plots the luminosity ratio, color-coded for spectral class, for each of the 575 candidates assuming each has an Earth-sized terrestrial planet at the mean $\mathrm{HZ}$. The ordinate is the number of stars with luminosity ratio greater than the value shown on the abscissa. The majority of star systems have luminosity ratios greater than $10^{-10}$ with most $\mathrm{A}$ stars having less favorable luminosity ratios. $\mathrm{G}, \mathrm{K}$ and $\mathrm{M}$ stars tend to have more favorable luminosity ratios. The luminosity ratio is independent of the telescope plus instrument and depends only on the star system.

Figure-6 plots the luminosity ratio for a planet in the HZ versus angular separation for each of the spectral classes. M and $\mathrm{K}$ stars tend to have more favorable luminosity ratios for detection; however, they tend to be colder stars with the $\mathrm{HZ}$ closer to the star requiring either a smaller IWA or larger aperture telescope to achieve the required contrast. $\mathrm{G}, \mathrm{F}$ and A stars have more favorable angular separations requiring larger IWA but smaller luminosity ratios requiring higher contrast to detect them. Figure-6 relates the telescope aperture (via the IWA) and the desired contrast (via the luminosity ratio) over the span of candidate stars in the HIP30 database. Figure-6 shows that the luminosity ratio scales as approximately the inverse of the angular separation independent of spectral class. This implies that a coronagraph may not have as stressing contrast requirements for $\mathrm{HZ}$ at smaller angular separations and a compromise exists between IWA and contrast when the available candidate stars are incorporated. 
The stellar photon count rate at the telescope aperture is estimated from the star's visual magnitude, aperture diameter and spectral bandpass. The stellar and terrestrial aperture photon count rate for a planet in the HZ, with $F_{0}=10^{8} \mathrm{ph} / \mathrm{sec}-m^{2}-n m$, are given by:

$$
\left\{\begin{array}{l}
F_{\text {STAR }}(p h / \mathrm{sec})=\pi F_{0} \times 10^{-\frac{M_{V}}{2.5}}(D / 2)^{2} \Delta \lambda \\
F_{\text {PLANET }}(p h / \mathrm{sec})=\left(L_{P} / L_{S}\right) \pi F_{0} \times 10^{-\frac{M_{V}}{2.5}}(D / 2)^{2} \Delta \lambda
\end{array}\right.
$$

Figure-7 plots the total collected of planetary photons/second for an aperture area of 1-meter ${ }^{2}$ assuming each candidate has a planet in the HZ with a $20 \%$ spectral filter of full-width-half-max (FWHM) of $\Delta \lambda=110 \mathrm{~nm}$ centered on $\lambda=550$.

The estimates are from applying the $2^{\text {nd }}$ equation in equation-4. Scaling to other aperture sizes is by multiplying by the aperture area in meters ${ }^{2}$. These aperture count rates are not the detector count rates since diffraction and scatter spread the counts out, and absorption losses in the telescope optics, instrument and detector reduce the focal plane count rates.

\subsection{Focal Plane Count Rates}

The photon counts incident upon the aperture are subsequently absorbed and diffracted in the optics, and, have imperfect conversion from photon to electron counts by the detector. The diffraction is a consequence of the finite aperture size and results in redistribution of the photons into an optical point spread function (PSF). The ideal PSF of a circular aperture is the Airy disk function, where $\theta=\sqrt{\theta_{x}^{2}+\theta_{y}^{2}}$ is the sky angle in units of lam/D, given by:

$$
\operatorname{PSF}(\theta)=\pi\left[\frac{J_{1}(\pi \theta)}{\pi \theta}\right]^{2} \approx \pi^{\frac{3}{2}} \frac{3 \sqrt{3}}{16} \frac{1}{1+\left(\pi^{4} / 8\right) \theta^{3}}
$$

The PSF is normalized such that its integral is unity. Its central core, to a radius of $\theta=1.22 \lambda / D$, contains $\sim 84 \%$ of the energy and a detector pixel is sized to account for $84 \%$ of the energy. 
The respective stellar and planetary energy are each distributed into separate PSFs centered on their sky locations. For an angular separation of $\theta_{P}$, in units of $\lambda / D$, the distribution of focal plane photoelectron count rates is given by:

$$
\left\{\begin{array}{l}
F_{\text {STAR }}(\theta)=0.84 S F_{0} 10^{-\frac{M_{V}}{2.5}}(D / 2)^{2}(3 \sqrt{3} / 16) \pi^{\frac{5}{3}}\left(1 / 1+\frac{\pi^{4}}{8} \theta^{3}\right) \Delta \lambda T_{o} q . e . \\
F_{\text {PLANET }}(\theta)=0.84\left(F_{P} / F_{S}\right) F_{0} 10^{-\frac{M_{V}}{2.5}}(D / 2)^{2}(3 \sqrt{3} / 16) \pi^{\frac{5}{3}}\left(1 / 1+\frac{\pi^{4}}{8}\left(\theta-\theta_{P}\right)^{3}\right) \Delta \lambda T_{o} q . e .
\end{array}\right.
$$

In equation-6 $T_{O}$ is the end-to-end transmission of the optics, q.e.is the quantum efficiency in units of photoelectrons/photon, and $\mathrm{S}$ is the starlight suppression factor of the coronagraph. The units of $F_{S T A R}(\theta)$ and $F_{\text {PLANET }}(\theta)$ are photoelectrons per pixel per second. Based on the above model the ratio of focal plane planet detector counts to star counts at the location of the planet $\theta=\theta_{P}$ is:

$$
Q\left(\theta_{P}\right)=\frac{F_{\text {PLANET }}\left(\theta_{P}\right)}{F_{\text {STAR }}\left(\theta_{P}\right)}=\frac{1}{S}\left(\frac{F_{P}}{F_{S}}\right)\left(1+\frac{\pi^{4}}{8} \theta_{P}^{3}\right)
$$

Where $Q$ is a function of the planet-to-star angular separation and since the model in equation- 6 has a $\theta_{P}^{3}$ dependency so does Q. All coronagraphs do not necessarily have this same dependency with angle but this is assumed based on assumptions about diffraction in Lyot and occulter type coronagraphs. It is likely that PIAA coronagraph, without an inverse PIAA, would have a higher order dependency, however in order to put the field of view back an inverse PIAA would be required and this would put the dependency back to $\sim \theta_{P}^{3}$. Nulling type coronagraphs perform beam nulling in the pupil plane but will ultimately be brought to focus with a limiting aperture introducing diffraction that is also $\sim \theta_{P}^{3}$.

Thus based on limited information herein we assume the $\theta_{p}^{3}$ but with the caveat that other dependencies are likely but 
are not yet well understood, as further information becomes available this should be revisited since a clear trade exists between the background diffraction dependency and performance of a given coronagraph.

Defining the contrast at the IWA as $C=S\left(1+\frac{\pi^{4}}{8} I W A^{3}\right)^{-1}$ gives:

$$
Q\left(\theta_{P}\right)=\frac{1}{C}\left(\frac{F_{P}}{F_{S}}\right) \frac{1+\frac{\pi^{4}}{8} \theta_{P}^{3}}{1+\frac{\pi^{4}}{8} I W A^{3}} \approx \frac{1}{C}\left(\frac{F_{P}}{F_{S}}\right)\left(\frac{\theta_{P}}{I W A}\right)^{3}
$$

Thus a star system with a planet in the $\mathrm{HZ}$ for a coronagraph at the IWA and operating at $\mathrm{C}=10^{10}$ would give $\mathrm{Q}=1$ implying the count rate from the star and planet in a pixel centered on the planet would be the same. "Q" expresses the ratio of the planet counts to leaked stellar counts and it is linear in contrast and linear in luminosity ratio but grows approximately as the cube of the angular separation when expressed in units of $\lambda / D$, implying doubling the aperture size moves the planet twice as far out in $\lambda / D$ units and thus gives an 8 fold gain in $\mathrm{Q}$. The optimal value of $\mathrm{Q}$ is discussed is a function of the sampling, detector noise characteristics, pointing jitter, finite size of a detectors pixel and where the planet occurs within the pixel, spectral bandpass and of the post-processing algorithms. Coronagraphs can operate with $Q<1$ since matched filtering, Bayesian estimation (Kasdin and Braems, (2006)) and nonlinear techniques can be employed to optimally estimate if a planet exists in a given dataset.

\subsection{Local and Exo-Zodiacal Light}

The above does not contain the effects of local zodiacal and exo-zodiacal light. Model and observations of exozodi generally show complex morphological behavior both radially and azimuthally and as function of the age of the stellar 
system (Absil, (2010), Stark and Kuchner (2008). Herein we are primarily concerned with the deleterious effects both Zodi and exoZodi would have on the detection of terrestrial planets and are thus express both the Zodi and exoZodi as simplified models only at the assumed location of a terrestrial planet. Zodi and exoZodi are usually expressed in magnitude per arcsecond square. Conversion to photoelectron count rate in a detector pixel of solid angle on the sky of $\Delta \Omega$ is given by:

$$
\left\{\begin{array}{l}
F_{A p: Z}=F_{0} 10^{-\frac{M_{Z}}{2.5}} \frac{\pi D^{2}}{4} \Delta \lambda \Delta \Omega T_{0} q . e . \\
F_{A p: E Z}=F_{0} 10^{-\frac{M_{E Z}}{2.5}} \frac{\pi D^{2}}{4} \Delta \lambda \Delta \Omega T_{0} q . e
\end{array}\right.
$$

The units of $\Delta \Omega$ are arcseconds-squared. Assuming that a given detector pixel is square of width $\lambda / D$ gives

$\Delta \Omega=(\lambda / D)^{2} *(3600 \times 180 / \pi)^{2} \operatorname{arcseconds}^{2}$ gives $\frac{\pi D^{2}}{4} \Delta \lambda \Delta \Omega=\frac{\pi}{4} \lambda^{2} \Delta \lambda *(3600 \times 180 / \pi)^{2}$ which is just

an expression of the radiance being conserved, i.e. the product of the aperture with solid angle is $\sim \lambda^{2}$.

Typical values of $M_{Z}=22$ magnitudes/arcsecond ${ }^{2}$ and $M_{E Z}=22$ magnitudes/arcsecond ${ }^{2}$ where this value of exoZodi is denoted as 1-Zodi. Using 22 magnitudes/aresec ${ }^{2}$ gives 0.15 photons/sec/pixel per meter ${ }^{2}$ of aperture without including the optics transmission and quantum efficiency. Figure -7 also shows the Zodi plotted as a solid line. Even 1 Zodi has the net effect that some of the planets yield lower count rates per pixel than the Zodi. While the exoZodi is not generally known models of it exist as a function of age of a given star system. Models show that Zodi is slowly varying spatial with respect to the scale of planet, hence it can in principle be subtracted off, but it does contribute photon noise after subtraction. Young systems, < 500 Myrs, can 1,000 's of times more dust than our solar system at 4.5 Gyrs age. The level of exoZodi represents a an unknown for the design of any high contrast exoplanet system at this time, however, ground based astronomy and JWST will likely make significant inroads to the levels of dust in nearby candidate systems. 


\subsection{Signal-to-Noise Ratio and Sensitivity}

The above set of calculations allows for the estimation of SNR parametrically as a function of aperture over the HIP30 database of candidate stars. With the above relations and SNR, in the form of sensitivity, we are in a better position to estimate the realizable science for a specific set of architectures and to error budget these architectures for various coronagraphic approaches. The SNR is given by:

$$
S N R=\frac{F_{P L A N E T} \Delta t}{\sqrt{F_{P L A N E T} \Delta t+F_{S T A R} \Delta t+F_{Z} \Delta t+F_{E Z} \Delta t+i_{D} \Delta t+\sigma_{e}^{2}}}
$$

$i_{D}$ is dark current in photoelectrons/sec/pixel, $\sigma_{e}$ is the detector readnoise and $\Delta t$ is the detector integration time. The residual speckle noise is implicitly included in the term $F_{\text {STAR }} \Delta t$ since this term is the noise variance of the residual leaked starlight due to incomplete starlight suppression and imperfect wavefront control. The term $F_{P L A N E T} \Delta t$ is the photon noise due to the planet, and $F_{Z} \Delta t$ and $F_{E Z} \Delta t$ are the photon noise assuming perfect subtraction of the Zodi and exoZodi. In practice these will be unlikely to be perfectly subtracted and will show up in a manner analogous to flat fielding error. The photon limited SNR can be taken as an upper bound on performance and is given by assuming that $\sigma_{e}=0$ and $i_{D}=0$ to give:

$$
S N R=\frac{F_{P L A N E T} \sqrt{\Delta t}}{\sqrt{F_{P L A N E T}+F_{S T A R}+F_{Z}+F_{E Z}}}
$$

The terms in the denominator are the photon noise from each the planet, stellar leakage (speckle noise), Zodi and exoZodi respectively. Scatter from out of field sources and background sources are not included. Solving equation-11 for time yields the photon limited time to a given SNR as: 


$$
\Delta t=S N R^{2}\left(\frac{F_{\text {PLANET }}+F_{\text {STAR }}+F_{Z}+F_{E Z}}{F_{\text {PLANET }}^{2}}\right)
$$

The terms within equation-12 were integrated over a V-band spectral filter for each of the 575 candidates for a 1 -meter diameter aperture and the "normalized" time versus angular separation plotted in Figure-8. The actual time (seconds) to SNR are given by dividing the value on the ordinate of Figure- 8 by the factor $D^{\frac{10}{3}}$ where $\mathrm{D}$ is the aperture diameter in units of meters. Thus, for example, a normalized time of $10^{6}$ seconds would scale down by a factor of $2^{\frac{10}{3}}=10.08$ or to $\sim 10^{5}$ seconds for a 2 -meter diameter aperture.

Times to different SNR's are given by multiplying by the ratio of squares of the SNRs per equation-12. Changing spectral filters is accomplished by multiplying time by the ratio of $110 \mathrm{~nm}$ to the new filters bandpass width - while this is an approximation it is reasonable in visible light.

Fig-8 is significant in that it couples the integration time for each of the candidates from each spectral class to its angular separation from the parent star for a planet in the HZ. The choice of aperture size in Figure- 8 has been left unconstrained since we desire to estimate the science return parametrically versus aperture, i.e. the scaling shown in Figure- 8 now allow for parametrically assessing exoplanet detection performance as a function of aperture size and these results are

Table-1: Candidate Stars versus Aperture

\begin{tabular}{|c|c|c|c|c|c|c|c|c|c|}
\hline \multirow{2}{*}{$\begin{array}{l}\text { Diameter } \\
\text { (meters) }\end{array}$} & \multirow{2}{*}{$\begin{array}{c}\text { IWA } \\
\text { (mas) }\end{array}$} & \multicolumn{7}{|c|}{ Number of Stars at or outside IWA } & \multirow{2}{*}{$\begin{array}{l}\Delta t \text { (days) } \\
\text { to } S N R=5\end{array}$} \\
\hline & & $A(18)$ & $F(27)$ & $G(124)$ & K (219) & $M(163)$ & $\mathrm{U}(24)$ & Total (575) & \\
\hline $1 \mathrm{~m}$ & 226.9 & $\overline{5}$ & $\overline{1}$ & $\overline{2}$ & 1 & $\overline{0}$ & $\overline{0}$ & $\overline{9}$ & 159.19 \\
\hline $2 \mathrm{~m}$ & 113.4 & 16 & 8 & 6 & 1 & 0 & 0 & 31 & 120.74 \\
\hline $4 \mathrm{~m}$ & 56.7 & 17 & 22 & 50 & 5 & 0 & 0 & 94 & 33.76 \\
\hline $8 \mathrm{~m}$ & 28.4 & 17 & 27 & 119 & 30 & 1 & 0 & 194 & 6.08 \\
\hline $16 \mathrm{~m}$ & 14.2 & 17 & 27 & 124 & 132 & 9 & 0 & 309 & 0.79 \\
\hline
\end{tabular}

shown in Table-1. 


\subsection{Detection Summary}

Table-1 summarizes the number of stars, that if exoplanets exist within their $\mathrm{HZ}$, could be detected for 5 aperture sizes; and, the amount of time to SNR=5 in days to detect these planets using a spectral filter centered on $550 \mathrm{~nm}$ with FWHM of $110 \mathrm{~nm}$. The number of stars increases with increasing aperture (Figure-9 top) size while the integration time decreases (Figure-9 bottom). A 4-meter aperture operating at 2 ND IWA would be capable of detecting a total of $94 \mathrm{HZ}$ exoplanets with 50 of those G-stars if exoplanets existed around them. If we assume that to completely search the system it would take 5 visits at 33.76 days per visit it would yield a total of 168.8 days and if $\eta_{\text {EARTH }}$ is $20 \%$ then potentially $\sim 18$ exoplanets in the $\mathrm{HZ}$ could be detected. At the time these 18 exoplanets were definitively detected they could be spectrally characterized to a $S N R=5$ with a $10 \mathrm{~nm}$ width spectral filter in $(110 \mathrm{~nm} / 10 \mathrm{~nm}) 33.8$ days $=371.4$ days, or approximately 1-year per planet. Lower spectral resolutions would give shorter times.

\subsection{Characterization and Spectroscopy}

Spectroscopic detection is one of the prime mission drivers since it is what would be used to determine if biomarkers existed within a planets atmosphere. Spectroscopy is more time consuming since the light must be dispersed into spectral bands and integrated long enough to achieve the SNR per spectral band. These times can be long if the collection area is small per the example in the previous section. Additionally the planet may not be visible throughout the time it takes to integrate enough light since the planet is moving in its orbit. Also the atmospheric abundances and pressures may be such that the absorption lines are only small dips in the spectrum and if not sufficiently pressured broadened could be very narrow, all of which when combined may make the spectral characterization difficult. An alternative approach is spectrophotometry, i.e. filter spectroscopy, whereby a set of filters centered on either specific spectral lines and/or colors are used. If the spectral filters are used serially then the times would still be long, however, if dichroic beamsplitters are used then all the filters could be brought to bear at the same time, effectively multiplexing the time as a dispersing spectrometer would, but with each filters width optimized for a specific spectral signature. While the range of options is

large and ill defined at this point we do show the time to SNR versus aperture size versus spectral resolution to enable setting a bound on the time to spectrally characterize. 


\section{ARCHITECTURE CONSIDERATIONS}

\subsection{Generic Model of Internal Coronagraph}

In order to assess the tolerances on a given architecture a generic approach is needed which applies to viable coronagraphs. In order to impart insight and delineate the sensitivities to errors and control frequencies an approximated analytic formalism is developed. An $8^{\text {th }}$ order Lyot-type coronagraph (Kuchner and Traub (2002)) is shown in Fig-10 whereby propagation of light through the coronagraph can be broken down to: (i) propagation from telescopes exit pupil to $1^{\text {st }}$ focal plane, (ii) application of an occulting mask, (iii) propagation from this occulting plane to a re-imaged exit pupil, (iv) application of a Lyot stop, and (v) propagation to the final science focal plane. While not immediately obvious, such a model can encompass most coronagraphs, provided the correct terms are identified. We will first step through the sequence of steps as shown in Fig-10 and subsequently approximate the terms that are important for wavefront and amplitude errors.

Let the complex electric field at the telescopes exit pupil be given by:

$$
P(\vec{r})=A(\vec{r})[1+\delta A(\vec{r})] e^{i \delta \phi(\vec{r})} \approx A_{0}[1+\delta A(\vec{r})+i \delta \phi(\vec{r})]
$$

Where the approximation is straightforward and based on retaining up to the $1^{\text {st }}$ order term in the small angle approximation of the phasor term. This is a common approximation in coronagraphy since in order for a coronagraph to operate at high contrast it must operate in a regime where the small angle approximation is valid. The effect of this approximation and its range of limitation has been explored in the context of coronagraphy by multiple authors see, Perrin et. al. (2003) and Sivaramakrishnan (2002).

The normalized pupil plane coordinates is given by $\vec{r}=(x, y)$ such that $|\vec{r}| \leq D / 2$ where $D$ is the diameter of the aperture. For elliptical or segmented or sparse apertures it is the largest distance between any two points within the aperture, $A_{0}$ is the mean amplitude and $\delta A(\vec{r})$ represents a fractional variation in the amplitude after traversing the telescope, $\delta \phi(\vec{r})$ is the phase errors incurred in passing through the telescope and $\delta \phi(\vec{r})=(2 \pi / \lambda) W(\vec{r})$ relates the wavefront error, $W(\vec{r})$, to the phase errors and where $\lambda$ is the wavelength. The errors in a coronagraph need to be 
small to detect a planet and hence the small angle approximation has been used in equation- 13 whereby terms to only first order have been retained.

Propagation through the sequence of 5 steps shown in figure- 10 is accomplished by stepping through:

$$
\left\{\begin{array}{l}
(i) \rightarrow \text { Exit pupil to focal plane } \\
(i i) \rightarrow \text { Apply occulting mask } \\
(i i) \rightarrow \text { Re-image the exit pupil } \\
(i v) \rightarrow \text { Apply Lyot stop } \\
(v) \rightarrow \text { Propagate to science focal plane }
\end{array}=\left\{\begin{array}{l}
A S F(\vec{\theta})=\int P(\vec{r}) e^{-i 2 \pi \frac{\vec{\theta} \cdot \vec{r}}{\lambda}} d \vec{r} \\
t_{C O R}(\vec{\theta}) A S F(\vec{\theta})=t_{C O R}(\vec{\theta}) \int P(\vec{r}) e^{-i 2 \pi \frac{\vec{\theta} \cdot \vec{r}}{\lambda}} d \vec{r} \\
\int t_{C O R}(\vec{\theta}) A S F(\vec{\theta}) e^{i 2 \pi \frac{\vec{\theta} \cdot \vec{r}}{\lambda}} d \vec{\theta}=\tilde{t}_{C O R}(\vec{r}) * * P(\vec{r}) \\
L(\vec{r})\left[\tilde{t}_{C O R}(\vec{r}) * * P(\vec{r})\right] \\
\int L(\vec{r})\left[\tilde{t}_{C O R}(\vec{r}) * * P(\vec{r})\right] e^{-i 2 \pi \frac{\tilde{\theta}^{2} \vec{r}}{\lambda}} d \vec{r}=A S F_{L}(\vec{\theta}) * *\left[t_{C O R}(\vec{\theta}) A S F(\vec{\theta})\right]
\end{array}\right.\right.
$$

In equation-14 $\vec{\theta}=\left(\theta_{x}, \theta_{y}\right)$ is the focal plane coordinate mapped to sky angular coordinates, $\operatorname{AS} F(\vec{\theta})$ is the complex scalar field in the $1^{\text {st }}$ focal plane known as the amplitude spread function, $t_{C O R}(\vec{\theta}) \in \square$ is the focal plane occulting mask and can either be real (amplitude mask) or complex (phase and/or amplitude and phase mask), $L(\vec{r})$ is the Lyot stop, and is usually a region of the re-imaged exit pupil that is smaller, i.e. less relative area, than the original exit pupil, and $A S F_{L}(\vec{\theta})$ is the amplitude spread function of the Lyot stop. "**" denotes 2D convolution. Magnifications are implicitly taken into account by choosing to work in relative pupil coordinates and angular sky coordinates. Overall phase factors have been neglected since they do not contribute the final result. The form of the propagators take the form of 2-dimensional Fourier transforms with conjugate variables given by $\vec{r}$ and $\vec{\theta}$. The science focal plane's complex electric field and intensity are respectively given by:

$$
\left\{\begin{array}{l}
E_{F}(\vec{\theta})=A S F_{L}(\vec{\theta}) * *\left(t_{C O R}(\vec{\theta}) A S F(\vec{\theta})\right) \\
I_{F}(\vec{\theta})=\left|A S F_{L}(\vec{\theta}) * *\left(t_{C O R}(\vec{\theta}) A S F(\vec{\theta})\right)\right|^{2}
\end{array}\right.
$$


Equation-15 describes the basic propagation through an internal coronagraph and can be used to model most variations including Lyot coronagraphs with various occulting masks and Lyot stops (Kuchner \& Traub, 2002), quadrant phase masks (Rouan 2000), vortex type coronagraphs (Mawet, 2010), and visible nulling coronagraphs (Lyon, 2011). External occulters (Cash 2006, Vanderbei 2007, Lyon, 2007) also approximately fit into this model since the starlight is suppressed prior to entering the telescope and the residual starlight entering the aperture is subsequently diffracted into an Airy like pattern. Phase Induced Amplitude Apodization (PIAA) (Guyon, 2003) performs pupil remapping and requires a different model. However, if a PIAA is used with an inverse PIAA the field of view is reconstructed after suppression and subsequently brought to focus yielding a similar dependence, i.e. the residual will follow approximately an Airy disk like dependence.

\subsection{Wavefront and Amplitude Errors}

The approach taken is to use the forms derived in section 3.1 based on the linear expansion of the wavefront phasor to assess first order requirements on sensing and control to achieve the contrast at the IWA for each of the candidate stars. Multiple authors have used various forms of these approaches in the context of coronagraphy to derive scaling relations (Soummer (2007), Sivaramakrishnan (2005)) as the linear approach imparts physical insight and is generally used for simplicity. Full scale modeling ultimately is required to assess where the deviations of the simpler models from the more rigorous models. The form we use herein is derived herein for completeness.

The approximated form of the telescope pupil can now be used in equation- 15 to yield:

$$
I_{F O C}(\vec{\theta}) \approx\left\langle\left|A S F_{L}(\vec{\theta}) * *\left\{t_{C O R}(\vec{\theta}) A S F(\vec{\theta})+t_{C O R}(\vec{\theta})\left\{A S F_{0}(\vec{\theta}) * *[\delta \tilde{A}(\vec{\theta})+i \delta \tilde{\phi}(\vec{\theta})]\right\}\right\}\right|^{2}\right\rangle
$$

where $A S F_{0}(\vec{\theta})$ is the amplitude spread function without amplitude and phase errors and $\delta \tilde{A}(\vec{\theta}), \delta \tilde{\phi}(\vec{\theta})$ are the 2D Fourier transforms of the amplitude and phase errors respectively. If $A S F_{0}(\vec{\theta})$ is much more compact than $\delta \tilde{A}(\vec{\theta})+i \delta \tilde{\phi}(\vec{\theta})$ then equation-16 can be approximated by:

$$
I_{F O C}(\vec{\theta}) \approx\left\langle\left|A S F_{L}(\vec{\theta}) * *\left\{t_{C O R}(\vec{\theta}) A S F(\vec{\theta})+t_{C O R}(\vec{\theta})[\delta \tilde{A}(\vec{\theta})+i \delta \tilde{\phi}(\vec{\theta})]\right\}\right|^{2}\right\rangle
$$


Let $\langle\delta \tilde{A}(\vec{\theta})\rangle=\langle\delta \tilde{\phi}(\vec{\theta})\rangle=0$, since mean amplitude and phase errors do not contribute to a lost in contrast, to give:

$$
I_{F O C}(\vec{\theta}) \approx P S F_{L}(\vec{\theta}) * *\left[T_{C O R}(\vec{\theta}) P S F(\vec{\theta})\right]+P S F_{L}(\vec{\theta}) * *\left[T_{C O R}(\vec{\theta})\left[P S D_{\delta A}(\vec{\theta})+P S D_{\delta \phi}(\vec{\theta})\right]\right]
$$

where $T_{C O R}(\vec{\theta})=\left|t_{C O R}(\vec{\theta})\right|^{2}$ is the intensity transmittance of the coronagraph versus angle on the sky and where we have used $P S D_{\delta A}(\vec{\theta})=\left\langle|\delta \tilde{A}(\vec{\theta})|^{2}\right\rangle$ and $\operatorname{PSD}_{\delta \phi}(\vec{\theta})=\left\langle|\delta \tilde{\phi}(\vec{\theta})|^{2}\right\rangle$ for the power spectral densities of the amplitude and phase errors respectively.

The power spectral densities are based on ensemble averages of the Fourier transforms of the wavefront and amplitude errors in keeping with the formal definition of the power spectral density. Thus a given PSD does not represent a specific realization of wavefront or amplitude induced speckle, but does represent an average over an ensemble of these speckles induced by different realizations of wavefront and amplitude errors. Use of PSDs to specify optical surfaces, wavefront and amplitude errors is now standardized approach and was used to specify the requirements on the Hubble Space Telescope, Chandra X-ray observatory (Harvey et.al (1988)) and the James Webb Space Telescope among others and a PSD transfer function approach was developed for a visible nulling coronagraph (Lyon (2005)).

A perfect coronagraph is when the first term, $P S F_{L}(\vec{\theta}) * *\left[T_{C O R}(\vec{\theta}) P S F_{0}(\vec{\theta})\right]=0$, and the remainder gives the leakage as:

$$
I_{L E A K}(\vec{\theta}) \approx P S F_{L}(\vec{\theta}) * *\left\{T_{C O R}(\vec{\theta})\left[P S D_{\delta A}(\vec{\theta})+P S D_{\delta \phi}(\vec{\theta})\right]\right\}
$$

If we normalize the PSDs such that integral over the focal plane, i.e. all spatial frequencies, is unity then the focal plane leakage is given by:

$$
I_{L E A K}(\vec{\theta}) \approx P S F_{L}(\vec{\theta}) * *\left\{T_{C O R}(\vec{\theta})\left[\sigma_{\delta A}^{2} P S D_{\delta A}(\vec{\theta})+\sigma_{\delta \phi}^{2} P S D_{\delta \phi}(\vec{\theta})\right]\right\}
$$


and $\sigma_{\delta A}^{2}, \sigma_{\delta \phi}^{2}$ are the amplitude and phase error variances respectively. where $\sigma_{\delta \phi}=\frac{2 \pi}{\lambda} \sigma_{\delta W}$ and $\sigma_{\delta W}$ is the rms wavefront error in the same units as the wavelength. Interchange between spatial frequencies, in cycles per aperture (cpa) and angle on the sky in units of $\lambda / D$, is trivial in these units; e.g. a spatial frequency of 4-cpa gives a speckle centered on $4 \lambda / D$ of width defined by the width of Lyot stop PSF, i.e. where $\mathrm{D}$ is the stopped down $\mathrm{D}$. The net effect is that, to $1^{\text {st }}$ order, amplitude and phase errors both induce focal plane speckle, however the focal plane location of the amplitude error induced speckle does not change nor scale with wavelength, whereas both the focal plane location and brightness of a wavefront error speckle varies with wavelength (Shaklan (2006)).

For a fixed given integral, i.e. a white noise and a power law PSD, both with the same power integrated out to a given spatial frequency, yields a power-law PSD with more power in the lower spatial frequencies thereby requiring progressively tighter tolerances on the sensing and control at low spatial frequencies and correspondingly less control at mid- and high-spatial frequencies. Low spatial frequencies arise from points on the surface that are far apart, i.e. long correlation lengths. Thus the most stressing tolerances are for those that are the most difficult to control.

A white noise PSD is however unlikely, conventional polishing and coating practices tend to yield mirror surface PSDs (Harvey, 2009, and Church, 1995) and residual coating error PSDs that follow power law functions of the form $\approx \frac{1}{1+\left(f / f_{n}\right)^{\alpha}}$ where $f_{n}$ is the knee spatial frequency, so called because on a log-log plot the PSD has break over point at the knee frequency. The exponent, $\alpha$, is typically or order $2-3$, and the point at which the PSD is larger than the PSF is the transition from diffraction to scatter limited (Bely 2003). If we assume a normalized, PSD of the form:

$$
\operatorname{PSD}(\vec{f})=\frac{\alpha}{2 \pi^{2} f_{n}^{2}} \sin \left(\frac{2 \pi}{\alpha}\right) \frac{\sigma_{T}^{2}}{1+\left(|\vec{f}| / f_{n}\right)^{\alpha}} \text { for } \alpha>2
$$

This functional form is normalized such that its integral over all 2D spatial frequencies is equal to the variance $\sigma_{T}^{2}$ of the total amplitude or phase error and where $|\vec{f}|=\sqrt{f_{x}^{2}+f_{y}^{2}}$. The PSD has units of power per spatial frequency squared, where spatial frequency in cpa or equivalently in $\lambda / D$ of sky angle. 


\subsection{Temporal Drift and Control}

The amplitude and phase errors are also dynamic in that they vary with time due to thermal and structural variations in the optics and the structures, which mount the optics. Even if the primary and secondary mirrors are dimensionally stable the truss structure has eigenmodes which when excited cause the footprint of the primary mirror (PM) beam to shift or shear on the secondary mirror (SM). This implies that the spatial frequency on the PM will shear on the SM causing temporal variations of both the amplitude and phase errors and this occurs throughout the optical system, i.e. modal excitations and thermal drift shift and distort the optical surfaces. Thus the PSDs have a component in the temporal frequency domain and are actually 3 -dimensional with respect to the frequencies $\left(f_{x}, f_{y}, f_{t}\right)$ where the first two frequencies are spatial frequencies in cpa in $x-$ and $-y$ directions and the third is the temporal frequency in $\mathrm{Hz}$. The 3D PSD then gives the power at a given spatial-temporal frequency. This is a more natural way to specify both the critical spatial and temporal frequencies, and the interplay between them, and lends itself well to the formalism of sensing and control via a transfer function approach for each contributor, and for wavefront and amplitude sensing and DM control.

Assume a temporal PSD of the form $\approx\left[1+\left(f_{t} / f_{D}\right)^{2}\right]^{-1}$ where $f_{D}$ is the temporal knee or drift frequency. It is likely that the underlying form of the temporal PSD approximately follows this functional form but with sharp or high Q modes due to the natural frequencies of the structure. Excitation of these modes can occur due to reaction and momentum wheels; the assumption herein is that they are well damped, or isolated, and that this PSD form represents a reasonable approximation to the underlying model.

The overall error spatial-temporal PSDs becomes:

$$
\operatorname{PSD}\left(\vec{f}, f_{t}\right)=\sigma_{T}^{2} \frac{\alpha}{\pi^{3} f_{n}^{2} f_{D}} \sin \left(\frac{2 \pi}{\alpha}\right) \frac{1}{1+\left(|\vec{f}| / f_{n}\right)^{\alpha}} \frac{1}{1+\left(f_{t} / f_{D}\right)^{2}} \text { for } \alpha>2
$$

with $f_{t} \in[0, \infty]$ and $|\vec{f}|=\sqrt{f_{x}^{2}+f_{y}^{2}}$ with $\left(f_{x}, f_{y}\right) \in[0, \infty]$. The units of the total error PSD are now power per spatial frequency squared per $\mathrm{Hz}$. The integral of total error PSD over all spatial and temporal frequencies is $\sigma_{T}^{2}$. There is an implicit assumption in this PSD form in that it assumes that the spatial errors are uncorrelated with the temporal 
errors. Strictly speaking this is not the most general case since for example a periodic temporal variation of the SM support structure will introduce time variations that are different at differing spatial frequencies. It is more proper to think of this separable functional form of the PSD as a bound on the errors.

Let the maximum frequency at which the deformable mirror (DM) is controlled be given by $f_{C}$, and let the spatial frequency exponent be $\alpha=3$ and divide the spatial frequency into the 3 bands for low-, mid- and high-spatial frequency. Integrating over the 3 bands from 0 to the control frequency with a knee frequency of $f_{n}=1 \mathrm{cpa}$ and $\alpha=3$ gives the contribution from each band:

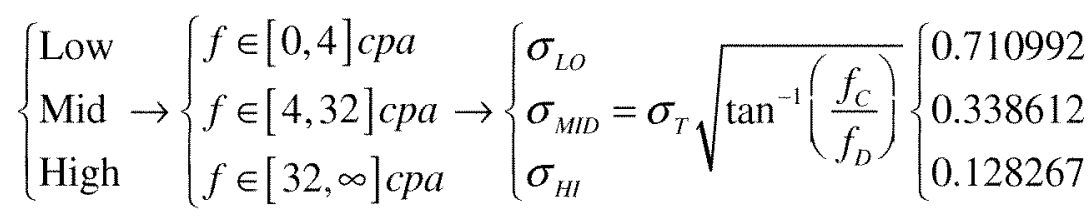

If $f_{C}>f_{D}$, or equivalently the system is stable relative to the control, then $\tan ^{-1}\left(f_{C} / f_{D}\right) \approx \pi / 2$ then $\sigma_{L O}^{2}+\sigma_{M I D}^{2}+\sigma_{H I}^{2}=\sigma_{T}^{2}$. Thus only as the ratio of the control to drift frequency exceeds unity the control efficacy approaches unity (Figure-11).

Folding in the temporal PSD leads to a modified leakage term by multiplying the PSDs by the idealized control term to give a leakage contribution in the science focal plane of:

$$
I_{L E A K}(\vec{\theta}) \approx \frac{\pi}{2} \frac{1}{\tan ^{-1}\left(f_{C} / f_{D}\right)} \operatorname{PSF}_{L}(\vec{\theta}) * *\left\{T_{C O R}(\vec{\theta})\left[\sigma_{\delta A}^{2} P S D_{\delta A}(\vec{\theta})+\sigma_{\delta \phi}^{2} P S D_{\delta \phi}(\vec{\theta})\right]\right\}
$$

Thus the leakage is strong function of the ratio of the control to drift frequency and for low control frequencies, $f_{C} \ll<f_{D}$, then $\frac{\pi}{2} \frac{1}{\tan ^{-1}\left(f_{C} / f_{D}\right)} \approx \frac{\pi}{2}\left(\frac{f_{D}}{f_{C}}\right)$ implies leakage increases linearly with drift frequency, i.e. as a random walk, where for $f_{C}>f_{D}$ the upper limit is asymptotically approached.

The ratio of the leaked starlight, at the planet location $\vec{\theta}_{P}$, to the planet light is given by $\frac{L_{S}}{L_{P}} I_{L E A K}\left(\vec{\theta}_{P}\right)$ where $\frac{L_{S}}{L_{P}}$ is the luminosity ratio of the star-to-planet. In general to 'see' the planet against this background requires 
$\frac{L_{P}}{L_{S} I_{L E A K}\left(\vec{\theta}_{P}\right)} \geq 1$, i.e. the planet should appear brighter than the leaked speckle. This implies that the fractional leaked starlight is at, or lower, than the luminosity ratio. The ratio $L_{S} / L_{P}$ is the inverse of the required operating contrast, therefore to 'see' the planet requires:

$$
I_{L E A K}\left(\vec{\theta}_{P}\right) \leq \frac{L_{P}}{L_{S}}=\frac{1}{\langle C\rangle}
$$

The required operating contrast $\langle C\rangle$ is set by the science and $I_{L E A K}\left(\vec{\theta}_{P}\right)$ relates it to the instrument requirements. Thus for each star with a planet in the $\mathrm{HZ}$ at an angular separation of $\vec{\theta}_{P}$, with a given luminosity ratio, a requirement can be set on $I_{L E A K}\left(\vec{\theta}_{P}\right)$ and thus a requirement on the rms amplitude and wavefront errors and implicitly on their PSDs.

Since the leakage is proportional to the inverse of the contrast this implies that the contrast increases with increasing control frequency, or conversely, that the tolerances on the amplitude and wavefront errors are more stressing for control frequencies slower than the drift frequencies. Thus a telescope with faster control, relative to its drift, has more relaxed tolerances on amplitude and wavefront errors.

If we assume a cubic spatial frequency power-law PSD, $\alpha=3$, and coronagraphic transmission at the IWA of $T(\theta)=1$ and integrating the leakage over a region defined by a focal plane speckle to give $I_{L E A K}(\vec{\theta}) \approx \sigma_{\delta \phi}^{2} P S D_{\delta \phi}(\vec{\theta})$ and integrating the $3 \mathrm{D}$ PSD out to the control frequency $f_{C}$ yields $\operatorname{PSD}(\vec{f})=\frac{3}{2 \pi^{2} f_{n}^{2}} \sqrt{\frac{3}{4}} \frac{\sigma_{T}^{2}}{1+\left(|\vec{f}| / f_{n}\right)^{3}}$. Equating the two gives:

$$
\begin{gathered}
\sigma_{W F E}=\frac{\lambda}{\sqrt{\langle C\rangle}} \frac{f_{n}}{3^{\frac{3}{4}}} \sqrt{\frac{2}{\pi} \tan ^{-1}\left(f_{C} / f_{D}\right)}\left[1+\left(|\vec{f}| / f_{n}\right)^{3}\right]^{\frac{1}{2}} \\
I_{L E A K}(\vec{\theta}) \approx \frac{\sigma_{T}^{2}}{\sqrt{3} \pi^{2}} \tan ^{-1}\left(\frac{f_{C}}{f_{D}}\right)=10^{-10} \text { and solving for } \sigma_{T}=3^{\frac{1}{4}} \pi \times 10^{-5} / \sqrt{\tan ^{-1}\left(\frac{f_{C}}{f_{D}}\right)} \text { where }
\end{gathered}
$$


to yield $\sigma_{W F E} \approx 0.0088652 \sqrt{\frac{2}{\pi} \tan ^{-1}\left(f_{C} / f_{D}\right)} \mathrm{nm}$,

where $\sigma_{W F E}$ is the rms wavefront error at a single spatial frequency of 2 cpa but with the temporal control folded in. If the control frequency is much greater than the drift frequency then $\frac{2}{\pi} \tan ^{-1}\left(f_{C} / f_{D}\right) \approx 1$ and an upper-bound of $\sigma_{W F E} \approx 0.0089 \mathrm{~nm}$ at $2 \mathrm{cpa}$. Note that this $\sigma_{W F E}$ is the rms of a single spatial term of the PSD integrated over all temporal frequencies. Figure-11 plots the wavefront error at this single spatial frequency versus the ratio of control to drift frequency. Thus, as expected, the tolerance to achieve and hold contrast is more stressing as the control frequency falls below the drift frequency. Control at frequencies less than the drift frequency places increasingly demanding tolerances on the wavefront and amplitude errors thus the lower the control frequency the lower the allowable errors and a clear trade exists between allowable wavefront error and the ratio of control to drift frequencies, and, this trade has significant impact on the design of a flight coronagraphic system.

For each of the candidate stars in the HIP30 database the expected HZ and flux ratio have been calculated (Figure-6) and this therefore sets a requirement on the wavefront and amplitude errors at the spatial frequency defined by the $\mathrm{HZ}$. A lower bound on the phase sensing precision is inferred from the Heisenberg uncertainty relations. The product of the uncertainty in photon counts and phase is given by $\langle n\rangle\langle\phi\rangle \geq 1 / 2$ and is equal to $1 / 2$ for all naturally occurring light (Loudon, 1983), and where $\langle n\rangle=\sigma_{n}$ and $\langle\phi\rangle=\sigma_{\phi}$. The lower bound on the standard deviation in sensing phase for a given photon rate is:

$$
\sigma_{\phi}=\frac{1}{2 \sqrt{n}}=\frac{1}{2 \sqrt{R_{n} \Delta t}} \rightarrow \sigma_{W F E}=\frac{\lambda}{4 \pi} \frac{1}{\sqrt{R_{n} \Delta t}}
$$

The number of detected photons is $n$, and $R_{n} \Delta t$ is the product of the photon count rate with integration time. This expresses a theoretical lower bound on the precision of phase sensing that in practice is usually not achievable due to sampling, detector quantization and other noise sources. However the theoretical precision can be used to estimate the bound on wavefront sensing precision. For example sensing of $0.1 \mathrm{~nm}$ rms wavefront error requires $\sim 200,000$ photons 
per speckle (or per spatial frequency). If only the photons that leak through the coronagraph are used for wavefront sensing then the integration times are longer than if all the photons could be optimally used.

To derive a theoretical bound for coronagraphic wavefront error use equations 21 and 27, and ignoring amplitude errors:

$$
\left.\operatorname{PSF}_{L}(\vec{\theta}) * *\left\{T_{C O R}(\vec{\theta})\left[\sigma_{\delta \phi}^{2} P S D_{\delta \phi}(\vec{\theta})\right]\right\}\right|_{\vec{\theta}=\vec{\theta}_{P}} \leq \frac{L_{P}}{L_{S}}=\frac{1}{\langle C\rangle}
$$

If we assume the integral (convolution) is taken over the region defined by a single speckle, i.e. over $P S F_{L}(\vec{\theta})$, and that the transmission of the coronagraph at the location of the planet is unity, i.e. $T_{C O R}\left(\vec{\theta}_{p}\right)=1$ and use the phase PSD with a knee frequency of 1-cpa and exponent of 3 and solve for the wavefront precision at the IWA gives:

$$
\sigma_{W F E}=\lambda \frac{3^{\frac{3}{4}}}{\pi \sqrt{2}} \frac{f_{n}}{\sqrt{\langle C\rangle}} \sqrt{\frac{2}{\pi} \tan ^{-1}\left(f_{C} / f_{D}\right)}\left[1+\left(I W A / f_{n}\right)^{3}\right]^{\frac{1}{2}}
$$

The wavefront error requirement derived in equation-29 scales inversely as the square root of contrast and approximately as the cube of the angular separation due to the assumed form for the wavefront error PSD. For a knee frequency such that $f_{n} \ll I W A$ the requirement scales as $\sim I W A^{\frac{3}{2}} / \sqrt{f_{n}}$ and for a knee frequency such $f_{n} \gg I W A$ (white noise assumption) the requirement scales linearly with the knee frequency.

Equation-29 expresses the required rms wavefront error required to achieve a given contrast at a given IWA folding in the knee frequency and ratio of control to drift frequency. The required rms wavefront error was estimated via equation29 for each of the HIP30 candidates and plotted versus angular separation in Figure-12. Figure-12 assumes a power law PSD with an exponent of 3 and a knee frequency of $1 \mathrm{cpa}$, a 4-meter telescope at a wavelength of $550 \mathrm{~nm}$, and assumes that the control frequency is fast with respect to the drift frequency.

The most stressing requirements are for the $\mathrm{G}$ stars and an angular separations of $1 \mathrm{lam} / \mathrm{D}$. The results for coronagraphs with IWA $=2$ lam/D does not give significantly different results yielding approximately wavefront error requirements of $\sim 0.006 \mathrm{~nm}$ rms. 
This requirement implicitly assumes the photons are available to sense to this level however there are clearly two cases: (1) all the photons from the target star, or, (2) only the photons that leak through the occulter, i.e. dark photons. Folding in the time to sense to each wavefront error requirement for each of the HIP30 stars under these two assumptions results in the two extremes plotted in Figure-13. Using only the dark photons implies only those stellar photons which are leaked by the occulting mask and Lyot stop, and using all the photons refers to using all the stellar photons collected by telescope. The time to sense the two extremes varies dramatically as shown in Figure-13.

A third case arises, that of internal metrology. Internal metrology would generally require photon sources internal to telescope and optics to sense the relative placements, deformations and drifts of the optics and components. In an internal metrology approach the photons used for sensing and control are not from the stellar source but from a source internal to the spacecraft, such as one or more lasers, and hence not limited by the stellar photon rates and could result in a robust sensing and control but with the added complexity of additional optics to route the lasers to the optics and possibly separate detectors. Such an approach should be considered as part of the sensing and control trade space but is not further discussed herein.

Figure-13 plots the time to sense to each of the required wavefront errors versus angular separation for each of the HIP30 candidates but assuming a V-band spectral filter centered on $550 \mathrm{~nm}$ with a 4-meter aperture. A broader filter would shorten the time, however, it has not yet been shown that sensing and control through a coronagraph is feasible with broadband filters. It is seen that the times are prohibitively long using only the leaked photons at the final control step. Such an approach would likely require internal metrology, unless as many of the photons that are available from all the photons collected are available, i.e. the photons which are reflected and/or absorbed at the occulting mask are also used (lower plot). Figure-13 assumes that effectively no drift occurs in the wavefront during the sensing time. If drift does occur then tolerances are more demanding and it would scale as the functional form shown in Figure-11.

One of the underlying questions is whether enough photons are collected from a given target star to control to the contrast needed? The results shown in Figure-13 attempts to answer that question. No reasonable mission could fly if only the leaked photons were used as the prime photon source for sensing and control. However, modulation schemes whereby the deformable mirror and/or other optics are deliberately moved to modulate the leaked photons would greatly shorten the time. A multitude of wavefront control approaches have been or under development and many are based on 
variations of electric field conjugation, see e.g. Give'on (2009) and references therein. What is not clear in these approaches are the amount of photons actually collected to achieve the contrast results.

Control can be broken down into a hierarchy of regimes. In optical sensing and control what is generally sensed is electrons from the detector from which it is inferred that photons are counted via the quantum efficiency, thus photon counts are measured and a model and/or algorithmic approach subsequently used to convert photons to estimates of the amplitude and wavefront errors. However, wavefront error cannot ever be directly measured since it corresponds to phase of the optical field which is not an observable (Loudon, 1983) and only photons converted to electrons in the form of current is measured.

Figure-14 shows an example of stability and control for the proposed Extrasolar Planetary Imaging Coronagraph (EPIC) mission (Clampin, 2009). Top left shows $\mathrm{rms}$ WFE in $\mathrm{nm}$ based on the rollup of a structural/thermal/optical model of the telescope and visible nulling coronagraph instrument versus time. Primary reason for growth in wavefront error is slow thermal drift due to spacecraft rolling with respect to sun angle during an observation from heliocentric orbit. The box shows the time window over which the requirement is met. Upper right shows the inverse of contrast (leakage) versus time. This is for a coronagraphic mission for Jovian planets and hence requires a less demanding $10^{9}$ contrast than a terrestrial planet mission. Based on the EPIC model a 7,000 sec stability window is achieved which allows for an active control scheme whereby null control sequences, $\sim 1,200 \mathrm{sec}$, are interlaced with observing sequences of approximately 7,000 seconds and where at the outset of observing sequence the wavefront error is better than required but drifts like a random walk up to its requirement prior to the null control sequence. A terrestrial planet detection mission will have more demanding tolerances on the achieving and maintaining the contrast which will likely drive it short stability times and hence short observation windows if an active scheme is used. An adaptive scheme where continuous control occurs through the science observation may be more robust.

For many optical control systems the phase is fit to the control modes of the DM, i.e. either as modeled or measured influence functions of the DM, or as a linear superposition of these influence functions, implying a linear model. Each influence function is the change in wavefront due to a unit motion of a single control degree-of-freedom (DOF) and there are as many influence functions as control DOF's. Each influence function is controlled by a voltage or set of voltages on the actuators. Various approaches such as combining the influence functions and solving the eigenmodes to 
orthogonalize and reduce the DOFs are available (Murphy 2001). However, from a control point of view these approaches infer wavefront and/or amplitude and subsequently decompose it into control modes, and feedback to actuators in either an active or adaptive fashion. An alternative approach is to directly map what is measured, i.e. photons (or electrons), to the control voltages of the DM and remove the step of converting to wavefront and amplitude errors, an approach deemed 'null control'. Such an approach implies that no separation of wavefront and amplitude errors are required, the only thing matters in a coronagraph are the leaked focal plane photons which reduce the contrast and thus the control system works to reduce these directly by choosing combinations of actuator motions which minimize the "darkness" at a specific or range of locations in the focal plane (Lyon (2011). This can also be performed in an active of adaptive fashion, where active refers to performing the control prior to each observation but not during it. If an out of specification condition occurred during the observation then control could be re-started. Adaptive refers to control throughout the observation in a closed-loop approach. There are advantages and disadvantages of each

Sensing and control defines a complex parameter space whereby square law type wavefront sensing (incoherent) versus interferometric (coherent) with using only the photons from the stellar source, or, using internal metrology. If the drift rates are fast relative to the photon collection time to sense for control then there may be no choice but to add internal metrology to mitigate the starved photon problem.

Derivations and assessment of the entire sensing and control trade space is outside the scope of this work but we attempted to address it at a conceptual level based upon expected stellar photon rates from the HIP30 catalog. The important parameter is the ratio of the drift to control rate; if the drift is slow with respect to the control then a quasistatic condition occurs whereby control may be needed only at the outset of an observation. One possibility would be to specify the telescope requirements such that this condition is always true, but it may result in telescope, which is either unbuildable or untestable, or prohibitively expensive. However, the telescope dynamic tolerances can only be relaxed to the point where the lowest photon rate science target still gives enough photons to achieve closed-loop. Otherwise a degradation in performance will result. Alternatively if only a few of the science sources drive the requirements, an alternative approach would be to remove those candidate sources deemed most stressing, alleviating the stressing requirements, or alternatively set the sensing and control separately for each star system such that it is optimal for the location of the $\mathrm{HZ}$. 


\section{SUMMARY AND CONCLUSIONS}

Herein we have mapped a candidate database of stars within 30 parsecs and deduced the flux ratio, contrast, inner working angle, and time to SNR, for each star for each spectral class, assuming an Earth-sized terrestrial planet in its habitable zone, and an IWA $=2 \lambda / \mathrm{D}$, and diffraction dependency on the background that scales as the cube of the inner working angle. Based on these assumptions the primary conclusions are:

(1) Assessment of all stars within 30 parsecs would require a 120-m telescope operating at IWA $=2 \lambda / \mathrm{D}$, however a 4-m telescope operating at IWA $==2 \lambda / \mathrm{D}$ would allow for searching 94 stars, of which 50 are G-stars, within $\sim 34$ days per visit, or $\sim 170$ days total if each was visited 5 times for completeness.

(2) The set of 575 candidate stars have flux ratios which vary from $3 \times 10^{-11}$ to $5 \times 10^{-8}$ with most G-stars having flux ratios between $3 \times 10^{-10}$ to $1 \times 10^{-8}$ implying contrast requirements at the IWA from $0.3 \times 10^{10}$ to $10^{8}$.

(3) The flux ratio has an approximately linear dependence versus planet-to-star angular separation with the majority of G-stars falling between 5 mas and 100 mas, however, the planets in closer in $\mathrm{HZ}$ have more favorable contrast ratios. $\mathrm{M}$-stars would have planets in the $\mathrm{HZ}$ and the smallest separation while $\mathrm{F}$ would be at the largest separations.

(4) The aperture flux of candidate planets in the $\mathrm{HZ}$ varies by only a factor of 3 over the span of angular separations and spectral classes.

(5) The $\mathrm{V}$-band sensitivity scales as $\sim D^{10 / 3}$ and yields time to $\mathrm{SNR}=5$ for a planet in the $\mathrm{HZ}$ that varies by approximately 4-orders of magnitude and for a 4-meter telescopes varies from $\sim 10$ seconds to $\sim 100,000$ seconds with most G-stars in the range of $1,000-100,000$ seconds.

(6) The luminosity ratio scales as approximately the inverse of the angular separation independent of spectral class implying that a clear trade/compromise exists between IWA and contrast when the available candidate stars are incorporated into the design approach.

(7) The most stressing stars, close IWA, requires wavefront tolerances of $\sim 0.008 \mathrm{~nm}$ rms wavefront error for control times that are comparable to drift times, however for slow control, i.e. control times that are long 
relative to the drift times requires significantly more demanding tolerances. Adaptive controls appears to be the most promising since this appears feasible relative to the dynamics of realistic spacecraft and wavefront drifts.

(8) The wavefront error requirements are a strong function of the angular separation and are $>0.008 \mathrm{~nm} \mathrm{rms}$ wavefront error for the majority of G-stars.

(9) The times to sense and control is limited by the photon statistics which sets a bound on recovery of wavefront errors based on the photon count rates. Wavefront or null control approaches based only the leaked starlight, without modulating to increase the counts, have prohibitively long sensing times. Modulation schemes, which deliberately change the wavefront errors in a known and deterministic way greatly increase the counts and hence shorten the sensing and control times. Approaches that use all the stellar photons have sensing times that are $\sim 8$-orders of magnitude shorter than schemes that use only leakage photons. In practice this can be accomplished with a visible nulling coronagraph that uses both the bright and dark output channels, or by approaches which pick stellar photons off from the front of the occulting mask.

\section{REFERENCES}

[1] Absil, O., Eiroa, C. Augereau, J.C., Beichman, C.A., Danchi, W.C., Defrere, D., Fridlund, M., Roberge, A., "Do we Need to Solve the Exozodi Question ? If Yes, How to Best Solve It?", Proc of Pathways Towards Habitable Planets, ASP Conference Series, Vol. 430, (2010)

[2] Bely, P.Y., "The Design and Construction of Large Optical Telescopes", Springer-Verlag, New York (2003)

[3] Brown, R.A., "Obscurational Completeness", ApJ 607:1003-1013, (2004)

[4] Brown, R.A., "Single-Visit Photometric and Obscurational Completeness", ApJ 624:1010-1024, (2005)

[5] Brown, R.A., and Soummer, R., "New Completeness Methods for Estimating Exoplanet Discoveries by Direct Detection", ApJ, 715:122-131, (2010)

[6] Cash, W., "Detection of Earth-like Planets around nearby Stars using a Petal-shaped Occulter", ApJ 738 (2006)

[7] Charbonneau, D., Noyes, R.W., Korzennik, S.G., Nisenson, P., Jha, S., "An Upper Limit on the Reflected Light from the Planet Orbiting the Star tau-Bootis," ApJ 522:L145-L148, (1999).

[8] Church, E.L. and Takacs, P.Z., "Light Scattering from Non-Gaussian Surfaces", Proc. SPIE 2541, 91-107 (1995). 
[9] Clampin, M., "Extrasolar Planetary Imaging Coronagraph (EPIC)", AAS Meeting \#213, \#234.06, BAAS Vol. 41, p 506, (2009)

[10]Clampin, M., Rieke, M., Rieke, G., Doyon, R., Krist, J., "Coronagraphic Detection of Exosolar Planets with the James Webb Space Telescope", http:/www.stsci.edu/jwst/science/whitepapers

[11]Fortney, J.J., Marley, M.S., Barnes, J.W., "Planetary Radii Across Five Orders of Magnitude in Mass and Stellar Insolatiion: Application to Transits," ApJ, 659:1661-1672, (2007).

[12] Give'on, A., "The Electric Field Conjugation: A Unified Formalism for Wavefront Correction Algorithms", OSA Adaptive Optics: Methods, Analysis and Application, San Jose CA, (2009)

[13] Guyon, O., "Phase-Induced Amplitude Apodization of Telescope Pupils for Extrasolar Terrestrial Planet Imaging", A\&A, 404:379-387 (2003)

[14] Harvey, J.E., Choi, N, Krywonos, A. and Marcen, J., "Calculating BRDFs from Surface PSDs for Moderately Rough Surfaces", Proc. SPIE 7426-42 (2009).

[15] Harvey, J.E., Moran, E.C., Zmek, W.P., "Transfer Function Characterization of Grazing Incidence Optical Systems", Applied Optics, Vol 27, (1988)

[16] Kasdin, N. J., and Braems, I., "Linear and Bayesian Planet Detection Algorithms for the Terrestrial Planet Finder", ApJ 646:1260-1274 (2006)

[17] Krist, J., Hartig, G., Clampin, M., Golimowski, D., Ford, H., Illingworth, G., “The Advanced Camera for Surveys Coronagraph on the Hubble Space Telescope," Proc. SPIE 4860 (2003).

[18] Kuchner, M.J, and Traub, W.A., "A Coronagraph with a Band-limited Mask for Finding Terrestrial Planets", ApJ, 570:900-908, (2002)

[19] Lallo, M. D., "Temporal behavior of HST: focus, coma and astigmatism history," Proc. SPIE 6270 (2006).

[20]Levine, M., Lisman, D., Shaklin, S., "Terrestrial Planet Finder Coronagraph Flight Baseline Mission Concept", hitp:/arxivorg/pdifo911.3200y (2009)

[21] Loudon, R., The Quantum Theory of Light, Second edition, Clarendon Press, Oxford, (1983)

[22] Lyon, R.G., Clampin, M., Woodruff R., Vasudevan, G., Shao, M., Levine, M., Melnick, G., Tolls, V., Petrone, P., Dogoda, P., Duval, J., Ge, J., "Visible Nulling Coronagraphy for Exo-Planetary Detection and Characterization", Proc of IAU No. 200, Direct Imaging of Exoplanets: Science and Technology, ed. C. Aime and F. Vakili, (2005) 
[23]Lyon, R.G., Woodruff, R.A., Brown, R. Noecker, M.C., Cheng, E., "Feasibility of Exoplanet Coronagraphy with the Hubble Space Telescope", Proc of SPIE 7731, San Diego CA, June 2010

[24]Lyon, R.G., Clampin, M., Petrone, P., Mallik, U., Madison, T., Bolcar, M.R., Noecker, M.C., Kendrick, S., Helmbrecht, M., "Vacuum Nuller Testbed Performance, Characterization and Null Control: Progress Report", Proc. of SPIE 8151, (2011)

[25]Lyon, R.G., Heap, S., Lo, A., Cash, W., Starkman, G.D., Vanderbei, R.J., Kasdin, N.J., Copi, C.J., "Externally Occulted Terrestrial Planet Finder Coronagraph: Simulations and Sensitivities", Proc of SPIE 6687, (2007)

[26] Mawet, D., Serabyn, E., Liewer, K., Burruss, R., Hickey, J. and Shemo, D., "The Vector Vortex Coronagraph: Laboratory Results and First Light at Palomar Observatory", ApJ, 709:53-57, (2010)

[27] Murphy, T.P., Lyon, R.G., Dorband, J.E. and Hollis, J.M., "Sparse Matrix Approximation Method for an Active Optical Control System", Applied Optics, 40, No. 35, (2001)

[28] Perrin, M., Sivaramakrishnan, A., Makidon, R.B., Oppenheimer, B.R., Graham, J.R., "The Structure of High Strehl Ratio Point-Spread Functions", ApJ 596: 702-712 (2003)

[29] Rouan, D., Riaud, P., Boccaletti, A., Clenet, Y., Labeyrie, A., "The Four-Quadrant Phase-Mask Coronagraph. I. Principle”, PASP, 112:1479-1486, (2000)

[30] Serabyn, E., Mawet, D., Burruss, R., "An Image of an Exoplanet Separated by Two Diffraction Beamwidths from a Star", Nature 464: 1018-1025 (2010)

[31] Shaklan, S.B., Green, J.J., Palacios, D.M., "The Terrestrial Planet Finder Coronagraph Optical Surface Requirements", Proc of SPIE Vol. 6265 (2006).

[32] Sivaramakrishnan, A., Lloyd, J.P., Hodge, P.E., Macintosh, B.A., "Speckle Decorrelation and Dynamic Range in Speckle Noise-Limited Imaging", ApJ, 581:L59-L62, (2002)

[33] Schneider, J., “The Extrasolar Planets Encyclopedia," htp:/lexoplanet.eu/, (2011)

[34] Soummer, R., Ferrari, A., Aime, C. and Jolissaint, L., "Speckle Noise and Dynamic Range in Coronagraphic Images", ApJ 669:642-656(2007)

[35] Trauger, J.T., and Traub, W. A., "A Laboratory Demonstration of the Capability to Image an Earth-like Extrasolar Planet", Nature 446: 771-773 (2007)

[36] Turon, C. et al, "Properties of the Hipparcos Input Cataloque", Astronomy \& Astrophysics, 304:82-93 (1995) 
[37] Vanderbei, R.J., Cady, E., Kasdin, N.J., "Optimal Occulter Design for Finding Extrasolar Planets", ApJ 665: 794798, (2007)

[38] Winn, J.N., "Transits and Occultations", arXiv:1001.2010v4

[39] Woodruff, R.A., Shao, M., Levine, B.M., Lyon, R.G., Vasudevan, G., Acu, J., Havey, K., Wynn, J., “Optical Design of Dilute Aperture Visible Nulling Coronagraph Imager (DAViNCI)", Proc of SPIE 7731 (2010) 


\section{FIGURE CAPTIONS}

Figure-1: Contrast and Focal Plane Diffraction. Example of a 4-meter circular aperture telescope's ideal diffraction pattern at $\lambda=500 \mathrm{~nm}$. The stars Airy disk normalized to unity at the peak (red) and a planet with a luminosity of $10^{-10}$ of the star separated by 100 mas (blue) would appear only $10^{6}-10^{7}$ times dimmer due to the angular separation.

Figure-2: Hipparcos Stars < 30 parsecs from Earth. All 2350 Hipparcos stars plotted versus distance from Earth (black) and then are filtered by selection criteria to a list of 575 candidates (blue) and separated by stellar spectral class.

Figure-3: Candidate Stars Mean Habitable Zone versus Distance to Star by Spectral Class. All candidate HZ's are $<1$ arcsec from parent star and concentrated between 1 and 100 milli-arcseconds.

Figure-4: HIP30 Stars mapped to Telescope Diameter. Candidate HZ is mapped to high contrast imaging system operating at IWA $=2 \lambda / \mathrm{D}$. Assessment of all stars within 30 parsecs would require a 120-m telescope, however, a 4-m would allow for searching 94 stars, of which 50 are G-stars, within $\sim 34$ days per visit, or $\sim 170$ days total if each was visited 5 times for completeness.

Figure-5: Number of Candidates versus Luminosity Ratio for each Spectral Class. Luminosity ratio is independent of the telescope and instrument and depends only on the star system.

Figure-6: Luminosity Ratio for Terrestrial Planet in $\mathrm{HZ}$ for each Spectral Class vs Angular Separation. Relates IWA and contrast to science requirements.

Figure-7: Aperture Flux of Planet in HZ vs Angular Separation. Zodi shown as flux in pixel of solid angle $\sim \lambda^{2}$.

Figure-8: Normalized $V$-band Sensitivity. Divide the normalized sensitivity by $\mathrm{D}^{10 / 3}$ to arrive at the actual time (in seconds) to achieve a SNR $=5$ for each candidate star with a planet in the $\mathrm{HZ}$ for a $\mathrm{V}$-band filter. 
Figure-9: (Top) Number of Stars $w / H Z$ at or outside IWA versus aperture diameter (bar plot of $2^{\text {nd }}$ to last column in Table-1). (Bottom) Time (days) to $S N R=5$ for a single observation of each of candidates versus aperture in Table-1 (last column of Table-1).

Figure-10: Star and Planet Light Propagation through $8^{\text {th }}$ Order Lyot Coronagraph

Figure-11: WFE at 2 cpa vs Control. Full control effectiveness is not reached until the control frequency exceeds the drift frequency.

Figure-12: Required WFE for each HIP3O candidate to yield stellar leakage, at the location of planet in $\mathrm{HZ}$, equal to the planet brightness assuming the control frequency is fast with respect to drift.

Figure-13: Photon Limited Time to sense WFE for each HIP30 candidate to yield leakage equal to planet brightness, at planet in $\mathrm{HZ}$, assuming fast control wrt the drift. Upper plot is time to sense assuming only photons that leak by the mask, in lower all photons are used.

Figure-14: Stability and Control Sequence for Extrasolar Planetary Imaging Coronagraph. Top left shows rms WFE in nm based on the rollup of a structural/thermal/optical model of the telescope and visible nulling coronagraph instrument versus time. Upper right shows the inverse of contrast (leakage) versus time. Bottom shows an active control scheme whereby null control sequences, $\sim 1,200 \mathrm{sec}$, are interlaced with observing sequences of approximately 7,000 seconds. 